\title{
Erratum to: High Glucose Induces Reactive Oxygen Species-Dependent Matrix Metalloproteinase-9 Expression and Cell Migration in Brain Astrocytes
}

\author{
His-Lung Hsieh • Chih-Chung Lin • Li-Der Hsiao • Chuen-Mao Yang
}

Published online: 19 April 2013

(C) Springer Science+Business Media New York 2013

Erratum to: Mol Neurobiol. 2013

DOI 10.1007/s12035-013-8442-6

For Figure 5-d, the authors made a mistake to present an error panel in zymogram which should be replaced with an exact one showed in this Corrigendum.
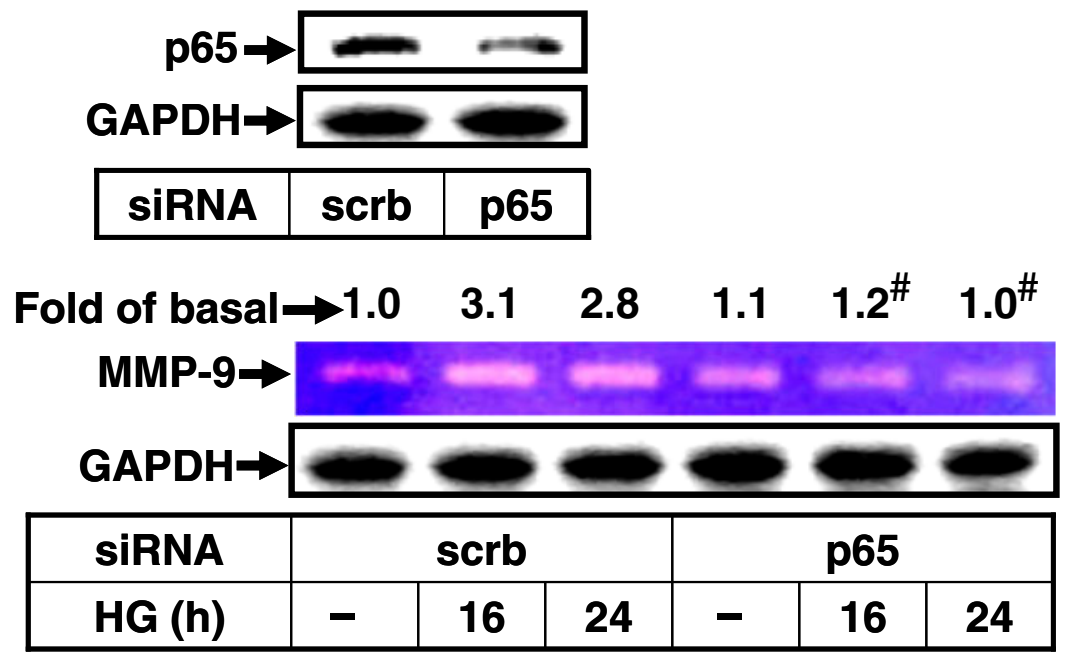

The authors regret the error.

The online version of the original article can be found at http://dx.doi/ org/10.1007/s12035-013-8442-6.

\section{H.-L. Hsieh}

Department of Nursing, Division of Basic Medical Sciences,

Chang Gung University of Science and Technology,

Tao-Yuan, Taiwan

\section{C.-C. Lin}

Department of Anesthetics, Chang Gung Memorial Hospital at Linkuo, and College of Medicine, Chang Gung University, Kwei-San,

Tao-Yuan, Taiwan

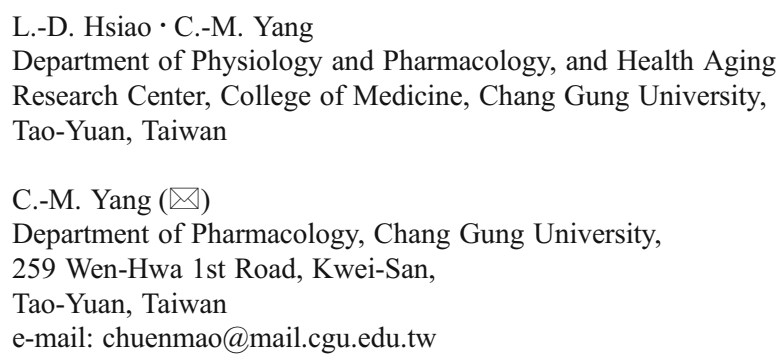

Department of Physiology and Pharmacology, and Health Aging Research Center, College of Medicine, Chang Gung University, Tao-Yuan, Taiwan

C.-M. Yang $(\bowtie)$

Department of Pharmacology, Chang Gung University,

259 Wen-Hwa 1st Road, Kwei-San,

Tao-Yuan, Taiwan

e-mail: chuenmao@mail.cgu.edu.tw 